\title{
Fuzzy Measures on Finite Scales as Families of Possibility Measures
}

\author{
Didier Dubois
}

\author{
IRIT, CNRS \& Université de Toulouse, France
}

\begin{abstract}
We show that any capacity or fuzzy measure ranging on a qualitative scale can be viewed both as the lower bound of a set of possibility measures, and the upper bound of a set of necessity measures. An algorithm is provided to compute the minimal set of possibility measures dominating a given capacity. This algorithm relies on the representation of the capacity by means of its qualitative Moebius transform, and the use of selection functions of the corresponding focal sets. We also introduce the counterpart of a contour function, that turns out to be the union of all most specific possibility distributions dominating the capacity. Finally we show the connection between Sugeno integrals and lower possibility measures.
\end{abstract}

Keywords: Fuzzy measures, possibility theory, qualitative Moebius transform.

\section{Introduction}

A fuzzy measure is a set-function that is monotonic under inclusion. If it takes value on a finite totally ordered scale, the usual Moebius transform cannot be properly defined. The qualitative counterpart of a Moebius transform has been introduced by Mesiar[12] and Grabisch [8] in 1997 and further studied by Grabisch [9]. It contains the minimal information from which a capacity can be reconstructed. This reconstruction process is similar to the way belief functions [13] are built from mass functions, replacing the sum by the maximum. On the other hand, the qualitative Moebius transform can also be viewed as a generalized possibility distribution, whereby a capacity is defined with respect to the latter by extending the definition of possibility measures. In fact the process of generation of belief functions, introduced by Dempster [2], was applied to possibility measures by Dubois and Prade $[5,6]$ so as to generate upper and lower possibilities and necessities. It was noticed that upper possibilities and lower necessities are still possibility and necessity measures respectively, but upper necessities and lower possibilities are not. A possibility distribution on one space gives rise to a basic possibility assignment on the other space. This study was pursued by Tsiporkova and De Baets [15] in a more general setting.
Since a mass function represents a family of probability measures that dominate the corresponding belief function, a natural question is whether a qualitative capacity can be viewed as a family of possibility measures, and if a qualitative Moebius transform can encode such a family. This paper tries to address this issue, taking up a pioneering work by Banon[1]. It shows that in the case of qualitative information, special subsets of possibility measures play a role similar to convex sets of probability measures. This should not come as a surprise. Indeed, it has been shown that possibility measures can be refined by probability measures using a lexicographic refinement of the basic axiom of possibility measures, and that capacities on a finite set can be refined by belief functions [4].

The paper is organized as follows. After recalling basic definitions about capacities, we show that any capacity can be viewed as a lower possibility measure. We lay bare a finite set of such possibility measures that are sufficient to characterize the capacity. Then, the inner qualitative Moebius transform is used to show that how to derive the minimal such set of possibility measures that dominate the capacity. We introduce the counterpart to the contour function of belief functions for capacities and show its relation to the minimal elements of the set of possibility measures dominating a capacity. We consider the dual construction using the outer qualitative Moebius transform and show that dually, a capacity can also be viewed as an upper necessity measure. Finally we show that Sugeno integral is a lower qualitative prioritised maximum in the sense of the possibility family generating the capacity.

\section{Fuzzy measures as Qualitative belief functions}

Consider a finite set $S$ and a finite totally ordered scale $L$ with top 1 and bottom 0 . A capacity (or fuzzy measure) is a mapping $\gamma: 2^{S} \rightarrow L$ such that

- $\gamma(\emptyset)=0$

- $\gamma(S)=1$

- If $A \subseteq B$ then $\gamma(A) \leq \gamma(B)$

A special case of capacity is a possibility measure. In possibility theory, the available information is represented by means of a possibility distribution. This is a function, usually denoted $\pi$, from the universe of discourse $S$ to the scale $L$. 
The function $\pi$ is supposed to rank-order potential values of (some aspect of) the state of the world - according to their plausibility. The value $\pi(s)$ is understood as the possibility that $s$ be the actual state of the world. Precise information corresponds to the situation where $\exists s^{*}, \pi\left(s^{*}\right)=1$, and $\forall s \neq s^{*}, \pi(s)=0$, while complete ignorance is represented by the vacuous possibility distribution $\pi^{\text {? }}$ such that $\forall s \in S, \pi^{?}(s)=1$. The possibility measure is defined by

$$
\Pi(A)=\max _{s \in A} \pi(s)
$$

A possibility distribution $\pi$ is said to be more specific than another possibility distribution $\rho$ if $\forall s \in$ $S, \pi(s) \leq \rho(s)$.

The inner (qualitative) Moebius transform of a capacity $\gamma$ is a mapping $\gamma_{\#}: 2^{S} \rightarrow L$ defined by

$$
\begin{aligned}
& \gamma_{\#}(E)=\gamma(E) \quad \text { if } \gamma(E)>\max _{B \subsetneq E} \gamma(B) \\
& =0 \quad \text { otherwise }
\end{aligned}
$$

In the above definition, due to the monotonicity property, the condition $\gamma(E)>\max _{B \subsetneq E} \gamma(B)$ can be replaced by $\max _{x \in E} \gamma(E \backslash\{x\})$. It is easy to check that

- $\gamma_{\#}(\emptyset)=0$;

- $\max _{A \subseteq S} \gamma_{\#}(A)=1$;

- If $A \subset B$, and $\gamma_{\#}(A)>0, \gamma_{\#}(B)>0$, then $\gamma_{\#}(A)<\gamma_{\#}(B)$.

Let $\mathcal{F}^{\gamma}=\left\{E, \gamma_{\#}(E)>0\right\}$ be the family of focal sets associated to $\gamma$. The last property says that the inner qualitative Moebius transform of $\gamma$ is strictly monotonic with inclusion on $\mathcal{F}^{\gamma}$.

It is clear that the inner qualitative Moebius transform of a possibility measure coincides with its possibility distribution: $\Pi_{\#}(A)=\pi(s)$ if $A=\{s\}$ and 0 otherwise. This property makes it clear that $\gamma_{\#}$ generalizes the notion of possibility distribution to the power set of $S$.

The inner (qualitative) Moebius transform contains the minimal information needed to reconstruct the capacity $\gamma$ since, by construction $[8,4]$ :

$$
\gamma(A)=\max _{E \subseteq A} \gamma_{\#}(E)
$$

The reader can check that if one of the values $\gamma_{\#}(E)$ is changed, the corresponding capacity will be different, namely the values $\gamma(A)$ such that $\gamma(A)=\gamma_{\#}(E)$.

The similarity between capacities and belief functions is striking on the above equation. A belief function is a set function $2^{S} \rightarrow[0,1]$ defined by

$$
\operatorname{Bel}(A)=\sum_{E, E \subseteq A} m(E), \forall A \subseteq S
$$

where $m$ is a mass function, i.e. a probability distribution over $2^{S} \backslash\{\emptyset\}$. The mass assignment $m$ can be reconstructed from $\mathrm{Bel}$ by inverting the system of equations: this is the Moebius transform [13]. In equation (1), max replaces the sum in the expression of Bel.

Hence function $\gamma_{\#}$ plays the role of a "qualitative" mass function obtained via a kind of Moebius transform [9]. The subsets $E$ that receive a positive support play the same role as the focal sets in Dempster-Shafer's theory: they are the primitive items of knowledge.

While an inner qualitative Moebius transform can be viewed as a basic possibility assignment on the power set of $S$, the converse is not true. Indeed given a possibility distribution $\pi$ on a space $\Omega$, and a multivalued mapping, $\Gamma: \Omega \rightarrow 2^{S}$, the possibilistic mass assignment $m_{\pi}(E)=\max _{\Gamma(\omega)=E} \pi(\omega)$ may fail to be satisfy the monotonicity constraint of a qualitative Moebius transform. It means that several basic possibility assignments may yield the same lower possibility via equation (1). If $m_{\pi}$ is a possibility distribution over $2^{S}$, monotonicity can be restored by deleting the terms $m_{\pi}(F)$ whenever $\exists E, E \subset F, m_{\pi}(E)>m_{\pi}(F)$. Indeed let $\gamma(A)=\max _{E \subseteq A} m_{\pi}(E)$. Clearly, if for some $F \subseteq A$, there is $E_{1} \subset F, m_{\pi}\left(E_{1}\right)>m_{\pi}(F)$, then $\gamma(A)=\max _{E \subseteq A, E \neq F} m_{\pi}(E)$. So we define a qualitative Moebius mass from $m_{\pi}$ as follows: $\gamma_{\#}(E)=$ $m_{\pi}(E)>0$ if and only if $\forall F \subset E, m_{\pi}(E)>m_{\pi}(F)$, otherwise $\gamma_{\#}(E)=0$.

In some sense, the inner qualitative Moebius transform is the canonical (monotonic) possibility assignment associated with the capacity $\gamma_{\#}$. Since any capacity can be expressed by a monotonic possibility assignment, a lower possibility is just any general capacity.

Note that the analogy between a capacity and a belief function does not mean that they have the same properties. Wong et al. [17] have shown that in order to be representable by a belief function, a qualitative capacity must satisfy the axiom

BEL: $\forall A, B, C$ disjoint, if $\gamma(A \cup B)>\gamma(A)$ then $\gamma(A \cup B \cup C)>\gamma(A \cup C)$.

Even if a capacity $\gamma$ may fail to satisfy axiom BEL, it almost satisfies it in the sense that the following property always holds :

$\forall A, B, C$ disjoint,

if $\gamma(A \cup B)>\gamma(A)$ then $\gamma(A \cup B \cup C) \geq \gamma(A \cup C)$.

It means that the weak ordering of events induced by any capacity can be refined by a weak ordering representable by a belief function [4].

It is well-known that a belief function can be equivalently represented by a convex set of probabilities. Let $\mathcal{P}(B e l)=\{P, P(A) \geq \operatorname{Bel}(A), \forall A \subseteq S\}$. Then it holds that $\operatorname{Bel}(A)=\min \{P(A): P \in$ $\mathcal{P}(\mathrm{Bel})\}$. In other words a belief function is one example of coherent lower probability in the sense of Walley [16] (exact capacity after Schmeidler [14]). 
In the qualitative case this construction makes no sense. The natural question is then whether a similar construction may make sense with qualitative possibility measures replacing probability measures.

\section{Capacities as Lower Possibilities}

There is always at least one possibility measure that dominates any capacity: the vacuous possibility measure, based on the distribution $\pi^{\text {? }}$ expressing ignorance, since then $\forall A \neq \emptyset \subset S, \Pi(A)=1 \geq$ $\gamma(A), \forall$ capacity $\gamma$, and $\Pi(\emptyset)=\gamma(\emptyset)=0$. Let

$$
\mathcal{R}(\gamma)=\{\pi: \Pi(A) \geq \gamma(A), \forall A \subseteq S\}
$$

be the set of possibility distributions whose corresponding set-functions $\Pi$ dominate $\gamma$. We call $\mathcal{R}(\gamma)$ the possibilistic credal set induced by the capacity $\gamma$. In this section we give some results on the structure of this set of possibility distributions.

Let $\sigma$ be a permutation of the $n=|S|$ elements in $S$. The $i$ th element of the permutation is denoted by $s_{\sigma(i)}$. Moreover let $S_{\sigma}^{i}=\left\{s_{\sigma(i)}, \ldots, s_{\sigma(n)}\right\}$. Define the possibility distribution $\pi_{\sigma}^{\gamma}$ as follows:

$$
\forall i=1 \ldots, n, \pi_{\sigma}^{\gamma}\left(s_{\sigma(i)}\right)=\gamma\left(S_{\sigma}^{i}\right)
$$

There are at most $n$ ! (number of permutations) such possibility distributions. It can be checked that the possibility measure $\Pi_{\sigma}^{\gamma}$ induced by $\pi_{\sigma}^{\gamma}$ lies in $\mathcal{R}(\gamma)$

Proposition $1 \forall A \subseteq S, \Pi_{\sigma}^{\gamma}(A) \geq \gamma(A)$.

Proof : Consider a given permutation $\sigma$ and an event $A$. Let $i_{A}=\max \left\{i, A \subseteq S_{\sigma}^{i}\right\}$. Then $S_{\sigma}^{i_{A}}$ is the smallest set in the sequence $\left\{S_{\sigma}^{i}\right\}_{i}$ that contains A. By construction, $\Pi_{\sigma}^{\gamma}\left(S_{\sigma}^{i_{A}}\right)=\gamma\left(S_{\sigma}^{i_{A}}\right) \geq \gamma(A)$. But $\Pi_{\sigma}^{\gamma}(A)=\max _{s \in A} \pi_{\sigma}^{\gamma}(s)=\max _{j=i, \ldots, n} \gamma\left(S_{\sigma}^{i}\right)=$ $\Pi_{\sigma}^{\gamma}\left(S_{\sigma}^{i_{A}}\right)=\gamma\left(S_{\sigma}^{i_{A}}\right)$ since $A$ contains $s_{\sigma\left(i_{A}\right)}$ and other elements in $A$ are of the form $s_{\sigma(j)}, j \geq i_{A}$ by construction.

More specifically the $n$ ! such possibility distributions enable $\gamma$ to be reconstructed (already in [1]):

Proposition $2 \forall A \subseteq S, \gamma(A)=\min _{\sigma} \Pi_{\sigma}^{\gamma}(A)$

Proof: Obviously, $\forall A \subseteq S$, there is a permutation $\sigma_{A}$ such that $A=S_{\sigma_{A}}^{i}$. By construction $\Pi_{\sigma_{A}}^{\gamma}(A)=\gamma(A)$. From the previous result, $\min _{\sigma} \Pi_{\sigma}^{\gamma}(A) \geq \gamma(A)$ and we see this infimum is attained by $\sigma=\sigma_{A}$.

As a consequence,

Proposition $3 \forall \pi \in \mathcal{R}(\gamma), \pi(s) \geq \pi_{\sigma}^{\gamma}(s), \forall s \in S$ for some permutation $\sigma$ of $S$.

Proof : Just consider a permutation $\sigma$ induced by $\pi$, that is $\sigma(i) \geq \sigma(j) \Longleftrightarrow \pi\left(s_{i}\right) \leq \pi\left(s_{j}\right)$. For this permutation, $\Pi\left(S_{\sigma}^{i}\right)=\pi\left(s_{i}\right) \geq \gamma\left(S_{\sigma}^{i}\right)=$ $\pi_{\sigma}^{\gamma}\left(s_{i}\right), \forall i=1, \ldots, n$.
This result says that the possibility distributions $\pi_{\sigma}^{\gamma}$ (we call the marginals of $\gamma$ ) include the least elements of $\mathcal{R}(\gamma)$ in the sense of fuzzy set inclusion, i.e., the most specific possibility distributions dominating $\gamma$. In other terms, $\mathcal{R}(\gamma)=\left\{\pi, \exists \sigma, \pi \geq \pi_{\sigma}^{\gamma}\right\}$. Of course the maximal element of $\mathcal{R}(\gamma)$ is the vacuous possibility distribution $\pi^{\text {? }}$.

These findings also show that any capacity can be viewed as a lower possibility measure. This is similar to the case of a belief function understood as a lower probability with respect to a (probabilistic) credal set $\mathcal{P}(\mathrm{Bel})$. This probability set forms a convex polyhedron whose vertices are among probability assignments $p_{\sigma}^{\gamma}$ of the form $p_{\sigma}^{\gamma}\left(s_{\sigma(i)}\right)=\operatorname{Bel}\left(S_{\sigma}^{i}\right)-\operatorname{Bel}\left(S_{\sigma}^{i+1}\right)$, and $\mathcal{P}(B e l)$ is the convex hull of these probabilities.

In the qualitative case, $\mathcal{R}(\gamma)$ is closed under the qualitative counterpart of a convex combination: if $\pi_{1}, \pi_{2} \in \mathcal{R}(\gamma)$, then $\forall \alpha, \beta \in$ $L$, such that $\max (\alpha, \beta)=1$, it holds that $\max \left(\min \left(\alpha, \pi_{1}\right),\left(\min \left(\beta, \pi_{2}\right)\right) \in \mathcal{R}(\gamma)\right.$. In fact $\mathcal{R}(\gamma)$ is an upper semi-lattice.

Conversely, given any set $\mathcal{T}$ of possibility distributions, the set-function $\gamma(A)=\min _{\pi \in \mathcal{T}} \Pi(A)$ is a capacity, and it is easy to see that $\mathcal{T} \subseteq \mathcal{R}(\gamma)$ and that if $\mathcal{T}$ contains possibility distributions that are not comparable with respect to specificity, $\mathcal{T}$ forms the most specific elements of $\mathcal{R}(\gamma)$. Note that the set function $\gamma(A)=\max _{\pi \in \mathcal{T}} \Pi(A)$ is not only a capacity, but also a possibility measure with possibility distribution $\pi^{\max }(s)=\max _{\pi \in \mathcal{T}} \pi(s)$. [7].

\section{From the Moebius transform to the possibilistic credal set}

In fact, not all $n$ ! possibility distributions $\pi_{\sigma}^{\gamma}$ are least elements of $\mathcal{R}(\gamma)$. As a trivial example, if $\gamma=\Pi$, this least element is unique and is precisely $\pi$. But other permutations yield other less specific possibility distributions. The aim of this section is to show that the qualitative Moebius transform is instrumental in finding these least elements.

\subsection{Selection functions}

We need the notion of a selection from a family $\mathcal{F} \subseteq 2^{S}$ of sets. A selection function sel $: \mathcal{F} \rightarrow S$ assigns to each subset $A \in \mathcal{F}$ an element $s=$ $\operatorname{sel}(A) \in A$. We denote by $\Sigma(\mathcal{F})$ the set of selection functions with domain $\mathcal{F}$. Given a capacity $\gamma$ and its inner qualitative Moebius transform $\gamma_{\#}$, consider a selection function sel whose domain is $\mathcal{F}^{\gamma}=\left\{E, \gamma_{\#}(E)>0\right\}$, the set of focal sets of $\gamma$. For any such selection function, one can define a possibility distribution $\pi_{\text {sel }}^{\gamma}$ by letting $\max \emptyset=0$ and

$$
\pi_{\text {sel }}^{\gamma}(s)=\max _{E: \operatorname{sel}(E)=s} \gamma_{\#}(E), \forall s \in S .
$$

Note that it parallels a similar construction in the theory of belief functions, where the probability assignments $p_{\text {sel }}^{B e l}(s)=\sum_{A: \operatorname{sel}(A)=s} m(A), \forall s \in S$ turn 
out to be the vertices of the credal set induced by Bel. In fact similar results for $\pi_{\text {sel }}^{\gamma}$ as for the $n$ ! possibility distributions $\pi_{\sigma}^{\gamma}$ can be obtained. Note that if $\gamma=\Pi$, then there is only one possible selection function and $\pi_{\text {sel }}^{\Pi}=\pi$, since $\mathcal{F}^{\gamma}$ only contains singletons (as recalled above eqn.(1)).

Proposition 4 For any selection function sel with domain $\mathcal{F}^{\gamma}$ it holds that $\forall A \subseteq S, \Pi_{\text {sel }}^{\gamma}(A) \geq \gamma(A)$.

Proof :

$$
\begin{aligned}
\Pi_{\text {sel }}^{\gamma}(A) & =\max _{s \in A} \pi_{\text {sel }}^{\gamma}(s)=\max _{s \in A} \max _{E: \operatorname{sel}(E)=s} \gamma_{\#}(E) \\
& \geq \max _{s \in A} \max _{E \subseteq A: \operatorname{sel}(E)=s} \gamma_{\#}(E)=\gamma(A)
\end{aligned}
$$

since if $E \subseteq A, \operatorname{sel}(E) \in A$ too, so $\{E \subseteq A: \exists s \in A: \operatorname{sel}(E)=s\}=\{E \subseteq A\}$.

In fact not all possibility distributions $\pi_{\text {sel }}^{\gamma}$ are of the form $\pi_{\sigma}^{\gamma}$ for a permutation $\sigma$.

Counterexample Suppose $S=\left\{s_{1}, s_{2}, s_{3}\right\}$, $\gamma_{\#}\left(\left\{s_{1}, s_{3}\right\}\right)=1, \gamma_{\#}\left(\left\{s_{2}, s_{3}\right\}\right)=\alpha<1$. Then consider $\operatorname{sel}\left(\left\{s_{1}, s_{3}\right\}\right)=s_{3}$ and $\operatorname{sel}\left(\left\{s_{2}, s_{3}\right\}\right)=s_{2}$, so that $\pi_{\text {sel }}^{\gamma}\left(s_{3}\right)=1>\pi_{\text {sel }}^{\gamma}\left(s_{2}\right)=\alpha>\pi_{\text {sel }}^{\gamma}\left(s_{1}\right)=0$. Using the corresponding permutation, note that $\pi_{\sigma}^{\gamma}\left(s_{3}\right)=1$ but $\pi_{\text {sel }}^{\gamma}\left(s_{2}\right)=\gamma\left(\left\{s_{1}, s_{2}\right\}\right)=0$. However if we choose another selection function sel $^{\prime}$ such that $\operatorname{sel}^{\prime}\left(\left\{s_{1}, s_{3}\right\}\right)=\operatorname{sel}^{\prime}\left(\left\{s_{2}, s_{3}\right\}\right)=s_{3}, \pi_{\text {sel }}^{\gamma}$ corresponds to the above $\pi_{\sigma}^{\gamma}$

Nevertheless we do have that the set $\left\{\pi_{\text {sel }}^{\gamma}\right.$, sel $\in$ $\left.\Sigma\left(\mathcal{F}^{\gamma}\right)\right\}$ also reconstructs $\gamma$.

Proposition $5 \forall A \subseteq S$,

$$
\gamma(A)=\min _{\operatorname{sel} \in \Sigma\left(\mathcal{F}^{\gamma}\right)} \Pi_{\text {sel }}^{\sigma}(A)
$$

Proof : $\Pi_{\text {sel }}^{\gamma}(A)=\max _{s \in A} \max _{E: \operatorname{sel}(E)=s} \gamma_{\#}(E)$. To get the equality, let $E \subseteq A$ be such that $\gamma(A)=\gamma_{\#}(E)$. Choose the selection function as follows: assign $\gamma_{\#}(E)$ to an element $s_{E} \in E$; then if $\gamma_{\#}(F)>\gamma_{\#}(E)$, assign $\gamma_{\#}(F)$ to some element not in $A$. This is possible, because if $\gamma_{\#}(F)>\gamma_{\#}(E)$, then $F \nsubseteq E$, and by assumption $F \nsubseteq A$ either. For such a selection function, $\Pi_{\text {sel }}^{\gamma}(A)=\pi_{\text {sel }}^{\gamma}\left(s_{E}\right)$ holds since the only elements in $A$ to which a possibility weight is assigned are $s_{E} \in E$, and possibly other $s_{C} \in C \cap A \neq \emptyset$, such that $\gamma_{\#}(C)<\gamma_{\#}(E)$. Hence $\Pi_{\text {sel }}^{\gamma}(A)=\gamma(A)$, due to Proposition 4 .

\subsection{Useful selection functions}

In fact the set of minimal elements (maximally specific) of $\mathcal{R}(\gamma)$ is also included in $\left\{\pi_{\text {sel }}^{\gamma}\right.$, sel $\left.\in \Sigma\left(\mathcal{F}^{\gamma}\right)\right\}$. Not all selection functions are of interest. For instance, if the set of focal sets of $\gamma$ has a common intersection $I$, and $\gamma_{\#}(E)=1$, if we let $\pi_{\text {sel }}^{\gamma}\left(s^{*}\right)=1$ for some $s^{*} \in I \subseteq E$ and $\pi_{\text {sel }}^{\gamma}(s)=\gamma_{\#}(F)<1$ for some $s \in F \backslash I$, the corresponding possibility distribution is not a most specific one, as the assignment of all $\gamma_{\#}(F)$ 's to the same element in the intersection of focal sets $I$ yields a more specific possibility distribution dominating $\gamma$, (having possibility 1 for this element and 0 to the rest, as in the above counterexample).

More generally the useful selection functions can be defined as follows.

Algorithm MSUP Maximal specific upper possibility generation

1. Rank the focal sets $E_{j}$ in decreasing order of $\gamma_{\#}\left(E_{j}\right)$. Let $j=1$ and $\mathcal{F}=\mathcal{F}^{\gamma}$.

2. Define $\operatorname{sel}\left(E_{j}\right)=s_{j}$ for some $s_{j} \in E_{j}$ and let $\pi\left(s_{j}\right)=\gamma_{\#}\left(E_{j}\right)$. Delete $E_{j}$ from $\mathcal{F}$.

3. $\forall k$ such that $s_{j} \in E_{k}$ for some other $E_{k} \in \mathcal{F}$ (it is such that $\left.\gamma_{\#}\left(E_{j}\right) \geq \gamma_{\#}\left(E_{k}\right)\right)$ let $\operatorname{sel}\left(E_{k}\right)=$ $s_{j}$, then delete $E_{k}$ from $\mathcal{F}$.

4. Repeat from step 2 until $\mathcal{F}=\emptyset$

In fact the above algorithm applies the trick in the proof of proposition 5 : the value $\gamma(A)=$ $\gamma_{\#}\left(E_{j}\right)$ is retrieved by a possibility distribution where $\gamma_{\#}\left(E_{k}\right), k<j$ are assigned outside $A$ (which is one option offered by the above procedure). A selection function sel thus generated satisfies the following property : If $\operatorname{sel}(E)=s$ for some $E \in \mathcal{F}^{\gamma}$ and $\pi_{\text {sel }}(s)=\gamma_{\#}(E)$, then $\forall F \in \mathcal{F}^{\gamma}$, such that $s \in F$ :

- if $\gamma_{\#}(E) \geq \gamma_{\#}(F)$ then $\operatorname{sel}(F)=s$

- if $\gamma_{\#}(E)<\gamma_{\#}(F)$ then $\operatorname{sel}(F) \neq s$.

Let $\Sigma_{*}(\gamma)$ be the set of selection functions generated by algorithm MSUP, called useful selection functions, and $\mathcal{R}_{*}(\gamma)$ be the corresponding set of possibility distributions. It can be checked that possibility distributions obtained in this way are not pairwise comparable, and that this family is enough to reconstruct the capacity.

It is clear that if sel $\in \Sigma_{*}(\gamma), \exists \sigma, \pi_{\text {sel }}=\pi_{\sigma}$. This is due to the ordering of elements generated by the procedure from great to small masses $\gamma_{\#}\left(E_{j}\right)$ : define $\sigma$ from the sequence of elements $s_{j}$ obtained by one application of the algorithm constructing sel. For $\pi_{\text {sel }}$, it is clear that $\pi_{s e l}\left(s_{\sigma(i)}\right) \leq$ $\pi_{\text {sel }}\left(s_{\sigma(j)}\right), \forall i>j$. Hence $\gamma\left(S_{i}^{\sigma}\right)=\Pi_{\text {sel }}\left(S_{i}^{\sigma}\right)$. Moreover, if two possibility distributions are generated by the algorithm, they correspond to different permutations (since each time, different elements receive positive masses).

So we conclude that $\mathcal{R}_{*}(\gamma)$ is a subset of $\left\{\pi_{\sigma}, \sigma\right.$ permutation of $S\}$.

Proposition 6 If $\pi \neq \rho \in \mathcal{R}_{*}(\gamma)$, then neither $\pi>$ $\rho$ nor $\pi>\rho$ hold.

Proof: Suppose $\pi>\rho$ generated by algorithm MSUP, i.e. $\pi \geq \rho$ and let $s$ be such that $\pi(s)>\rho(s)$. Let $s e l_{1}$ and $s e l_{2}$ be the selection functions associated with $\pi$ and $\rho$, respectively. Let $E$ be such that $\operatorname{sel}_{1}(E)=s$ and $\pi(s)=\gamma_{\#}(E)>\rho(s)$. By construction, $\operatorname{sel}_{2}(E)=s^{\prime} \neq s$. Then $\rho\left(s^{\prime}\right) \geq$ 
$\gamma_{\#}(E)>\rho(s)$. So, $\exists F, \gamma_{\#}(F) \geq \gamma_{\#}(E)$ such that sel $_{2}(F)=s^{\prime}$ and $\rho\left(s^{\prime}\right)=\gamma_{\#}(F)$. Since $\pi\left(s^{\prime}\right) \geq$ $\rho\left(s^{\prime}\right), \exists G, \gamma_{\#}(G) \geq \gamma_{\#}(E)$ such that $\operatorname{sel}_{1}(G)=s^{\prime}$ and $\pi\left(s^{\prime}\right)=\gamma_{\#}(G)$. But since by construction, $s^{\prime} \in$ $E \cap G$, algorithm MSUP would enforce $\operatorname{sel}_{1}(E)=s^{\prime}$, which contradicts the assumption that $\operatorname{sel}_{1}(E)=s$. So $\pi \geq \rho$ with $\pi(s)>\rho(s)$ is in conflict with the assumption that $\pi$ comes from the algorithm.

Proposition 7 For any permutation $\sigma$, there exists a selection function sel corresponding to another permutation $\tau$ such that $\pi_{\sigma} \geq \pi_{\tau}=\pi_{\text {sel }}$.

Proof: Let $\sigma$ be a permutation of $S$, and we denote the corresponding elements as $s_{i}$ for simplicity. Let $\pi\left(s_{i}\right)=\gamma\left(S_{i}\right)$ where $S_{i}=\left\{s_{i}, \ldots, s_{n}\right\}$. Let us show that we can derive a useful selection function from $\sigma$. For each $s_{i}$ we shall decide whether not to make it a selection from $E \in \mathcal{F}^{\gamma}$. Let $U$ be the set of undecided elements, and $\mathcal{F}$ the current set of focal sets.

- Let $U=S$ and $\mathcal{F}=\mathcal{F}^{\gamma}$. If $\pi_{\sigma}\left(s_{j}\right)=0$ for $j=i, \ldots, n$, the elements $s_{j}$ must be discarded from $S$ and will not be selected from focal sets. Set $U=:\left\{s_{1}, \ldots s_{i-1}\right\}$.

- For $j=i-1$ down to 1

- Select $E \in \mathcal{F}$ such that $s_{j} \in E, E \subseteq S_{j}$, $\gamma_{\#}(E)$ is maximal, and let $\operatorname{sel}(E)=s_{j}$. If there is none, let $\pi_{\text {sel }}\left(s_{j}\right)=0$. Delete $s_{j}$ from $U$

- let $\operatorname{sel}(F)=s_{j}$ for all $F$ such that $s_{j} \in F$ and $F \subseteq S_{j}$ and $\gamma_{\#}(F) \leq \gamma_{\#}(E)$, and delete such $F$ from $\mathcal{F}$.

- Set $j=: j-1$

It is clear that the obtained selection function sel is useful. Moreover, it is also obvious that $\pi_{\text {sel }}\left(s_{i}\right) \leq \pi\left(s_{i}\right)$ since $\pi_{\text {sel }}\left(s_{i}\right)=\gamma_{\#}\left(E_{i}\right)$ for some $E_{i} \subseteq S_{i}$. Now if we use the permutation $\tau$ such that $\pi_{\text {sel }}\left(s_{\tau}(i)\right) \leq \pi_{\text {sel }}\left(s_{\tau}(j)\right)$ whenever $i>j$, then we have that $\pi_{\text {sel }}=\pi_{\tau}$ (this is because $\pi_{\text {sel }}\left(s_{\tau}(i)\right)<\pi_{\text {sel }}\left(s_{\tau}(j)\right)$ if and only if $\left.\gamma_{\#}\left(E_{i}\right)<\gamma_{\#}\left(E_{j}\right)\right)$.

\section{Example}

In the following we denote sets by concatenation of letters. Consider the set abcdef. Let $\gamma$ be such that $\gamma_{\#}(a b)=1>\gamma_{\#}(b c)=0.8>\gamma_{\#}(e f)=0.7>$ $\gamma_{\#}(c d e)=0.6>\gamma_{\#}(a f)=0.5$.

Generation of a useful selection by MSUP: choose $\operatorname{sel}(a b)=a$. It enforces $\operatorname{sel}(a f)=a$. Then choose $\operatorname{sel}(b c)=b$, then choose $\operatorname{sel}(e f)=e$, and it enforces $\operatorname{sel}(c d e)=e$. So, $\pi_{\text {sel }}(a)=1 ; \pi_{\text {sel }}(b)=$ $0.8 ; \pi_{\text {sel }}(e)=0.7$; and $\pi_{\text {sel }}(s)=0$ otherwise.

Generation of all useful selections by MSUP: The following selection functions can be found using this procedure:

1. $\operatorname{sel}(a b)=a, \operatorname{sel}(b c)=b, \operatorname{sel}(e f)=e, \operatorname{sel}(c d e)=$ $e, \operatorname{sel}(a f)=a$; then $\pi_{\text {sel }}(a)=1, \pi_{\text {sel }}(b)=$ $0.8, \pi_{\text {sel }}(e)=0.7$; and $\pi_{\text {sel }}(s)=0$ otherwise.
2. $\operatorname{sel}(a b)=a, \operatorname{sel}(b c)=b, \operatorname{sel}(e f)=f, \operatorname{sel}(c d e)=$ $c, \operatorname{sel}(a f)=a$; then $\pi_{\text {sel }}(a)=1, \pi_{\text {sel }}(b)=$ $0.8, \pi_{\text {sel }}(c)=0.6 ; \pi_{\text {sel }}(f)=0.7 ;$ and $\pi_{\text {sel }}(s)=0$ otherwise.

3. $\operatorname{sel}(a b)=a, \operatorname{sel}(b c)=b, \operatorname{sel}(e f)=f, \operatorname{sel}(c d e)=$ $d, \operatorname{sel}(a f)=a ;$ then $\pi_{\text {sel }}(a)=1, \pi_{\text {sel }}(b)=$ $0.8, \pi_{\text {sel }}(d)=0.6, \pi_{\text {sel }}(f)=0.7$; and $\pi_{\text {sel }}(s)=$ 0 otherwise.

4. $\operatorname{sel}(a b)=a, \operatorname{sel}(b c)=b, \operatorname{sel}($ ef $)=f, \operatorname{sel}($ cde $)=$ $e, \operatorname{sel}(a f)=a$; then $\pi_{\text {sel }}(a)=1, \pi_{\text {sel }}(b)=$ $0.8, \pi_{\text {sel }}(e)=0.6, \pi_{\text {sel }}(f)=0.7$; and $\pi_{\text {sel }}(s)=0$ otherwise.

5. $\operatorname{sel}(a b)=a, \operatorname{sel}(b c)=c, \operatorname{sel}(e f)=e, \operatorname{sel}(c d e)=$ $c, \operatorname{sel}(a f)=a$; then $\pi_{\text {sel }}(a)=1, \pi_{\text {sel }}(c)=$ $0.8, \pi_{\text {sel }}(e)=0.7$, and $\pi_{\text {sel }}(s)=0$ otherwise.

6. $\operatorname{sel}(a b)=a, \operatorname{sel}(b c)=c, \operatorname{sel}(e f)=f, \operatorname{sel}(c d e)=$ $c, \operatorname{sel}(a f)=a$; then $\pi_{\text {sel }}(a)=1, \pi_{\text {sel }}(c)=$ $0.8, \pi_{\text {sel }}(f)=0.7$, and $\pi_{\text {sel }}(s)=0$ otherwise.

7. $\operatorname{sel}(a b)=b, \operatorname{sel}(b c)=b, \operatorname{sel}(e f)=e, \operatorname{sel}(c d e)=$ $e, \operatorname{sel}(a f)=a$; then $\pi_{\text {sel }}(a)=0.5, \pi_{\text {sel }}(b)=$ $1, \pi_{\text {sel }}(e)=0.7$, and $\pi_{\text {sel }}(s)=0$ otherwise.

8. $\operatorname{sel}(a b)=b, \operatorname{sel}(b c)=b, \operatorname{sel}(e f)=e, \operatorname{sel}(c d e)=$ $e, \operatorname{sel}(a f)=f ;$ then $\pi_{\text {sel }}(b)=1, \pi_{\text {sel }}(e)=$ $0.7, \pi_{\text {sel }}(f)=0.5$, and $\pi_{\text {sel }}(s)=0$ otherwise.

9. $\operatorname{sel}(a b)=b, \operatorname{sel}(b c)=b, \operatorname{sel}($ ef $)=f, \operatorname{sel}(c d e)=$ $c, \operatorname{sel}(a f)=f ;$ then $\pi_{\text {sel }}(b)=1, \pi_{\text {sel }}(c)=$ $0.6, \pi_{\text {sel }}(f)=0.7$, and $\pi_{\text {sel }}(s)=0$ otherwise.

10. $\operatorname{sel}(a b)=b, \operatorname{sel}(b c)=b, \operatorname{sel}(e f)=f, \operatorname{sel}(c d e)=$ $d, \operatorname{sel}(a f)=f ;$ then $\pi_{\text {sel }}(b)=1, \pi_{\text {sel }}(d)=$ $0.6, \pi_{\text {sel }}(f)=0.7$, and $\pi_{\text {sel }}(s)=0$ otherwise.

11. $\operatorname{sel}(a b)=b, \operatorname{sel}(b c)=b, \operatorname{sel}(e f)=f, \operatorname{sel}(c d e)=$ $e, \operatorname{sel}(a f)=f$. then $\pi_{\text {sel }}(b)=1, \pi_{\text {sel }}(e)=$ $0.6, \pi_{\text {sel }}(f)=0.7$, and $\pi_{\text {sel }}(s)=0$ otherwise.

Generation of a useful selection from a permutation. Consider the permutation $\sigma=f e d c b a$ in this order. It is clear that $\pi_{\sigma}\left(S_{i}\right)=1, \forall i<6$ and $\pi_{\sigma}(a)=0$. This possibility distribution is a very loose upper bound of $\gamma$. Let us find a more informative permutation via a selection:

1. First we can delete $a$ from $U$ and $\pi_{\text {sel }}(a)=0$.

2. Then we select $E=a b$ and $\operatorname{sel}(a b)=b$. We also let $\operatorname{sel}(b c)=b$. Now $U=\operatorname{cdef}$ and $\mathcal{F}=$ $\{e f, c d e, a f\}$

3. We can select no focal set in $a b c$, nor in $a b c d$. So $\pi_{\text {sel }}(c)=\pi_{\text {sel }}(d)=0$. Now $U=e f$

4. Then we select $E=c d e \subset$ abcde and sel(cde) $=$ e. Now $U=f$ and $\mathcal{F}=\{e f, a f\}$

5. Finally select $E=$ ef and let $\operatorname{sel}(e f)=$ $\operatorname{sel}(a f)=f$.

By construction, $\pi_{\text {sel }}(b)=1, \pi_{\text {sel }}(e)=$ $0.6, \pi_{\text {sel }}(f)=0.7$ and 0 otherwise. It corresponds to several permutations, for instance $c d a e f b$, where indeed $\Pi_{s e l}($ bfeadc $)=\pi_{\text {sel }}(b)=1$, $\Pi_{s e l}($ feadc $)=\pi_{\text {sel }}(f)=0.7, \Pi_{\text {sel }}($ eadc $)=$ $\pi_{s e l}(e)=0.6, \Pi_{s e l}(a d c)=\Pi_{s e l}(d c)=\Pi_{s e l}(c)=0$.

Note that while not all selection functions $\pi_{\text {sel }}$ yield a permutation $\sigma$ such that $\pi_{s e l}=\pi_{\sigma}$, this is true for useful selection functions. Now we are in a 
position to show that useful selection functions provide a minimal set of possibility distributions that are enough to represent the possibilistic credal set of a capacity $\gamma$.

Proposition $8 \mathcal{R}_{*}(\gamma)$ is the set of maximally specific possibility distributions such that $\Pi(A) \geq$ $\gamma(A)$.

Proof: Suppose $\pi \in \mathcal{R}(\gamma)$. From Proposition 3, there is a permutation $\sigma$ of $S$ such that $\pi \geq \pi_{\sigma}$. Moreover there is a useful selection function sel such that $\pi_{\sigma} \geq \pi_{\text {sel }}$. Since possibility distributions constructed from a useful selection function are not comparable, they form the minimal elements of $\pi \in \mathcal{R}(\gamma)$.

\section{Remarks}

- Note that if $\gamma_{\#}(E)>0, \forall E \neq \emptyset \subseteq S, \mathcal{R}_{*}(\gamma)$ contains all $\pi_{\sigma}$ for all permutations, since at each round of the algorithm, it is possible to choose any remaining element and assign the weight $\gamma_{\#}(E)>0$ to it for some focal set $E$.

- If $\gamma$ is a necessity measure $N$, such that $N(A \cap B)=\min (N(A), N(B))$ then its qualitative Moebius transform is a collection of nested sets $E_{1} \subset E_{2} \subset, \ldots E_{k}$ such that $N(A)=\max _{E_{i} \subseteq A} N_{\#}\left(E_{i}\right)$. The useful selection functions are then such that if $\operatorname{sel}\left(E_{i}\right)=s$, $\operatorname{sel}\left(E_{k}\right) \neq s$ for $k>i$ and $\operatorname{sel}\left(E_{k}\right)=s$ if $s \in E_{k}, k>i$. In particular one may choose $\operatorname{sel}\left(E_{i}\right)=s \in E_{1}, \forall E_{i}$. But a necessity measure is based on a simple possibility distribution $\pi$ such that $N(A)=\min _{s \notin A} \nu(\pi(s))$, where $\nu$ is the order-reversing map on $L$. So it is not worth approximating $N$ from above by a family of possibility distributions.

\subsection{Qualitative contour functions}

It is possible to introduce the counterpart of a contour function (for belief functions, it is $\mu: S \rightarrow[0,1]$ defined by $\mu(s)=\sum_{s \in E} m(E)$. Namely, define

$$
\pi_{\gamma}(s)=\max _{s \in E} \gamma_{\#}(E)
$$

to be the qualitative contour function of $\gamma$. It can be proved that

Proposition $9 \pi_{\gamma}(s)=\max _{\pi \in \mathcal{R}_{*}(\gamma)} \pi(s)$. Moreover, $\forall A, \Pi_{\gamma}(A)=\max _{A \cap E \neq \emptyset} \gamma_{\#}(E)$

\section{Proof : Indeed,}

$\max _{s e l \in \Sigma(\gamma)} \pi_{\text {sel }}(s)=\max _{\text {sel } \in \Sigma(\gamma), \operatorname{sel}(E)=s} \gamma_{\#}(E)$ and

$\{E: \operatorname{sel}(E)=s, \forall s e l \in \Sigma(\gamma)\}=\{E: s \in E\}$.

We can restrict to useful selections, since $\forall E, \gamma_{\#}(E)>0$, there is a useful selection such that $\operatorname{sel}(E)=s$ and $\pi_{\text {sel }}(s)=\gamma_{\#}(E)$.

Note that $\{E: A \cap E \neq \emptyset\}=\{E, \exists s \in A \cap E\}$. And $\Pi_{\gamma}(A)=\max _{s \in A} \max _{s \in E} \gamma_{\#}(E)$.
Clearly, the situation of qualitative capacities with respect to possibility measures is different from the one of numerical capacities with respect to probabilities in some respects:

- Any qualitative capacity is a lower possibility, but not any numerical capacity is a lower probability.

- The qualitative counterpart to a plausibility function is a possibility function that dominates the capacity, and its possibility distribution is the qualitative contour function of the capacity, the union of all the most specific possibility distributions that dominate it.

If we consider the set of possibility measures generated by a possibility distribution via a multivalued mapping, yielding an upper possibility function $\bar{\Pi}$ and a lower possibility function $\underline{\Pi}=\gamma$ then $\bar{\Pi}=\Pi_{\gamma}$ and the set of so-generated possibility functions is $\left\{\pi, \gamma(A) \leq \Pi(A) \leq \Pi_{\gamma}(A), \forall A \subseteq S\right\}$ and not just the set of possibility functions dominating $\gamma$. However, we cannot use the necessity measure induced by the contour function of $\gamma$ as a lower bound for the latter. Indeed $N_{\gamma}(A)=\nu\left(\Pi_{\gamma}\left(A^{c}\right)\right)=$ $\min _{s \notin A} \nu\left(\max _{s \in E} \gamma_{\#}(E)\right)=\min _{E \cap A^{c} \neq \emptyset} \nu\left(\gamma_{\#}(E)\right)$, which cannot be compared with $\gamma(A)$.

\section{Capacities as upper necessities}

In order to directly refine a necessity measure, another qualitative representation of a capacity $\gamma$ can be used, namely a new set function, denoted $\gamma^{\#}$, the knowledge of which is again enough to reconstruct the capacity:

$$
\begin{aligned}
\gamma^{\#}(A) & =\gamma(A) \text { if } \gamma(A)<\min \{\gamma(F), A \subset F\} \\
& =1 \text { otherwise. }
\end{aligned}
$$

In particular, $\gamma^{\#}(S)=1$. Due to the monotonicity of $\gamma$, the condition $\gamma(A)<\min \{\gamma(F), A \subset F\}$ can be equivalently replaced by $\gamma(A)<\min \{\gamma(A \cup$ $\{s\}), s \notin A\}$.

The original capacity is then retrieved as [4]:

$$
\gamma(A)=\min _{A \subseteq F} \gamma^{\#}(F)
$$

which reminds of outer measures. Function $\gamma^{\#}$ can be called outer qualitative mass function of $\gamma$, as $\gamma(A)$ is recovered from $\gamma^{\#}$ via weights assigned to supersets of set $A$, while $\gamma_{\#}$ stands for an inner qualitative mass function.

Denote by $\gamma^{c}$ the conjugate of $\gamma$, defined as $\gamma^{c}(A)=\nu\left(\gamma\left(A^{c}\right)\right), \forall A \subseteq S$, where $A^{c}$ is the complement of set $A$, and $\nu$ the order-reversing map on $L$. The inner qualitative mass function $\gamma_{\#}^{c}$ of $\gamma^{c}$ is related to the outer qualitative mass function $\gamma^{\#}$ [4]:

$$
\gamma^{\#}(E)=\nu\left(\gamma_{\#}^{c}\left(E^{c}\right)\right) .
$$

since $\gamma(A)<\min \{\gamma(F), A \subset F\}$ also writes $\gamma^{c}\left(A^{c}\right)>\max \left\{\gamma^{c}\left(F^{c}\right), F^{c} \subset A^{c}\right\}$. For instance in 
the case of a necessity measure $N^{\#}(E) \neq 1$ only if $E=S \backslash\{s\}$ for some $s \in S, \pi(s)>0$, and then $N^{\#}(S \backslash\{s\})=\nu(\pi(s))$. The necessity measure is then of the form

$$
N(A)=\min _{s \notin A} N^{\#}(S \backslash\{s\})=\nu\left(\max _{s \notin A} \pi(s)\right) .
$$

This expression can be generalized to any capacity $\gamma$ by rewriting (6) as follows:

$$
\gamma(A)=\nu\left(\max _{A \cap F=\emptyset} \gamma_{\#}^{c}(F)\right),
$$

in terms of the inner Moebius transform of the conjugate. So we can describe capacity functions as upper necessities by means of a family of necessity functions that stem from the lower possibility description of their conjugates. So, the inner Moebius transform is the basic information from which we can derive a capacity and its conjugate. Then we can define two sets of possibility functions from $\gamma$ :

- The set $\mathcal{R}(\gamma)$ of possibility measures that dominate $\gamma$;

- The set $\mathcal{R}\left(\gamma^{c}\right)$ of possibility measures that dominate its conjugate $\gamma^{c}$;

Both sets of possibility measures can be defined from selection functions applied to the focal sets of $\gamma$ and its conjugate, respectively. In each case we can restrict to useful selection functions. Clearly, possibility measures that dominate $\gamma^{c}$ are conjugates of necessity measures dominated by $\gamma$. In other words $\gamma$ is also an upper necessity measure in the sense that

$$
\gamma(A)=\max \left\{N(A), \pi \in \mathcal{R}_{*}\left(\gamma^{c}\right)\right\} .
$$

We can denote the set of minimal possibility distributions generating maximal necessity measures under $\gamma$ by $\mathcal{R}^{*}(\gamma)=\mathcal{R}_{*}\left(\gamma^{c}\right)$. Likewise, the contour function of $\gamma^{c}$ can serve as a lower "impossibility distribution", namely consider the contour function of $\gamma^{c}$, that is, $\pi_{\gamma_{c}}(s)=\max _{s \in E} \gamma_{\#}^{c}(E)$, and define an "anti-contour" function in the form of an impossibility distribution $\iota_{\gamma}: S \rightarrow L$ as $\iota_{\gamma}(s)=\nu\left(\pi_{\gamma_{c}}(s)\right)=N_{\gamma_{c}}(S \backslash\{s\})$. Then it can be checked that

$$
\gamma(A) \geq N_{\gamma_{c}}(A)=\min _{s \notin A} \iota_{\gamma}(s) .
$$

Moreover $N_{\gamma_{c}}(A)=\min _{A^{c} \cap E \neq \emptyset} \nu\left(\gamma_{\#}^{c}(E)\right)$ defines a lower necessity measure that is a necessity measure with possibility distribution $\pi_{\gamma_{c}}$.

One representation of $\gamma$ by means of $\mathcal{R}_{*}(\gamma)$ or $\mathcal{R}_{*}\left(\gamma^{c}\right)$ may be simpler that the other. For instance, if $\gamma$ is a necessity measure based on possibility distribution $\pi$, then $\mathcal{R}^{*}(\gamma)=\{\pi\}$ while $\mathcal{R}_{*}(\gamma)$ contains several possibility distributions including $\pi$. Note that $\Pi(A) \geq N(A)=\Pi^{c}(A)$, so that it looks more natural to reach $N$ from below and $\Pi$ from above. More generally if a capacity $\gamma$ is such that $\gamma(A) \geq \gamma^{c}(A), \forall A \subseteq S,(\gamma$ is an upper capacity) then it is clear that $\mathcal{R}_{*}(\gamma) \subset \mathcal{R}\left(\gamma^{c}\right)$ is more natural than $\mathcal{R}_{*}\left(\gamma^{c}\right)$ for representing $\gamma$ by a family of possibility measures that dominate it.

\section{Sugeno integrals as lower possibilistic expectations}

Let $f: S \rightarrow L$ be a function that may serve as a utility function if $S$ is a set of attributes. Sugeno integral is often defined as follows:

$$
\mathcal{S}_{\gamma}(f)=\max _{\lambda \in L} \min \left(\lambda, \gamma\left(F_{\lambda}\right)\right)
$$

where $F_{\lambda}=\{s: f(s) \geq \lambda\}$ is the set of attributes having best ratings for some object, above threshold $\lambda$, and $\gamma(A)$ is the degree of importance of feature set $A$. An equivalent expression is [10]:

$$
\mathcal{S}_{\gamma}(f)=\max _{A \subseteq S} \min \left(\gamma(A), \min _{s \in A} f(s)\right)
$$

In this disjunctive form, the set-function $\gamma$ can be replaced without loss of information by the inner qualitative Moebius transform $\gamma_{\#}$ defined earlier.

$$
\mathcal{S}_{\gamma}(f)=\max _{A \in \mathcal{F}^{\gamma}} \min \left(\gamma_{\#}(A), f_{A}\right)
$$

where $f_{A}=\min _{s \in A} f(s)$. The above expression of Sugeno integral has the standard maxmin form, viewing $\gamma_{\#}$ as a possibility distribution over $2^{S}$.

When $\gamma$ is a possibility measure $\Pi$, Sugeno integral in form (9) simplifies into:

$$
\mathcal{S}_{\Pi}(f)=\max _{s \in S} \min (\pi(s), f(s))
$$

which is the prioritized max. It can be proved that Sugeno integral is a lower prioritized maximum:

Proposition $10 \mathcal{S}_{\gamma}(f)=\inf _{\pi \in \mathcal{R}_{*}(\gamma)} \mathcal{S}_{\Pi}(f)$

Proof: Viewing $\gamma$ as a lower possibility, it comes: $\mathcal{S}_{\gamma}(f)=\max _{A \subseteq S} \min \left(\min _{\pi \in \mathcal{R}_{*}(\gamma)} \Pi(A), f_{A}\right)$

$=\max _{A \subseteq S} \min _{\pi \in \mathcal{R}_{*}(\gamma)} \min \left(\Pi(A), f_{A}\right)$

$\leq \min _{\pi \in \mathcal{R}_{*}(\gamma)} \max _{A \subseteq S} \min \left(\Pi(A), f_{A}\right)$.

hence $\mathcal{S}_{\gamma}(f) \leq \inf _{\pi \in \mathcal{R}_{*}(\gamma)} \mathcal{S}_{\Pi}(f)$

Conversely, let $\pi_{f}$ be the marginal of $\gamma$ obtained from the nested sequence of sets $F_{\lambda}$ induced by function $f$, then it is clear that $\Pi_{f}\left(F_{\lambda}\right)=\gamma\left(F_{\lambda}\right)$, and thus $\mathcal{S}_{\gamma}(f)=\mathcal{S}_{\Pi_{f}}(f)$. As $\exists \pi \in \mathcal{R}_{*}(\gamma), \pi_{f} \geq \pi$, by definition, $\mathcal{S}_{\Pi_{f}}(f) \geq \mathcal{S}_{\Pi}(f) \geq \inf _{\pi \in \mathcal{R}_{*}(\gamma)} \mathcal{S}_{\Pi}(f)$.

The obtained equality may sound surprizing. If $\gamma=\min \left(\gamma_{1}, \gamma_{2}\right)$, it only holds in general that $\mathcal{S}_{\min \left(\gamma_{1}, \gamma_{2}\right)}(f) \leq \min \left(\mathcal{S}_{\gamma_{1}}(f), \mathcal{S}_{\gamma_{2}}(f)\right)$. Nevertheless, equality holds if $\gamma=\min \left(\Pi_{1}, \Pi_{2}\right)$, for two possibility measures, that is $\mathcal{R}_{*}(\gamma)=\left\{\pi_{1}, \pi_{2}\right\}$. This is because any possibility measure $\Pi \geq \gamma$ is such that $\pi \geq \pi_{1}$ or $\pi \geq \pi_{2}$. To see it directly, suppose first that $\pi\left(s_{1}\right)<\pi_{1}\left(s_{1}\right)$ and $\pi\left(s_{2}\right)<\pi_{2}\left(s_{2}\right)$ for some $s_{1}, s_{2} \in S$. Then $\Pi\left(\left\{s_{1}, s_{2}\right\}\right)<\gamma\left(\left\{s_{1}, s_{2}\right\}\right)$, which violates the assumption $\Pi \geq \gamma$. So, $\forall s \in S, \Pi \geq \gamma$ implies either $\pi(s) \geq \pi_{1}(s)$ or $\pi(s) \geq \pi_{2}(s)$. Now suppose $\pi_{2}\left(s_{1}\right)>\pi\left(s_{1}\right) \geq \pi_{1}\left(s_{1}\right)$. Then if for some other $s \neq s_{1}, \pi(s)<\pi_{1}(s)$, again $\Pi\left(\left\{s_{1}, s\right\}\right)<$ $\gamma\left(\left\{s_{1}, s\right\}\right)$. Hence, $\pi \geq \pi_{1}$. So, $\mathcal{S}_{\gamma}(f)=\mathcal{S}_{\Pi_{f}}(f) \geq$ $\min \left(\mathcal{S}_{\Pi_{1}}(f), \mathcal{S}_{\Pi_{2}}(f)\right)$ since $\Pi_{f} \geq \gamma$. 
Note that in the numerical case, the same feature occurs, namely, lower expectations with respect to a convex probability set are sometimes Choquet integrals with respect to the capacity equal to the lower probability constructed from this probability set (for instance convex capacities, and belief functions). However, this is not true for any capacity and any convex probability set.

Finally, using conjugacy properties, one could prove that $\mathcal{S}_{\gamma}(f)=\sup _{\pi \in \mathcal{R}_{*}\left(\gamma^{c}\right)} \mathcal{S}_{N}(f)$ as well.

\section{Conclusion}

We have studied the representation of capacities having values on a finite totally ordered scale by families of qualitative possibility distributions. We could prove that any capacity can be viewed as a lower possibility measure and an upper necessity measure with respect to two distinct families of possibility distributions. A procedure exploiting the qualitative Moebius transform of the capacity has been given to enumerate the most specific possibility measures dominating a capacity. The number of such possibility measures can actually vary from 1 to $n$ ! where $n$ is the number of elements in the set. We also introduced the counterpart of a contour function for qualitative capacities. Note that Grabisch [8] introduced the qualitative counterpart of a Shapley value. Finally we show Sugeno integrals are both lower prioritized minima, hence, by conjugacy, upper prioritized maxima.

This study takes a point of view opposite to previous works generating upper and lower possibilities and necessities $[6,6,15]$. We show that lower possibilities and upper necessities cover the whole family of capacities, since all qualitative Moebius transforms can be obtained from a possibility distribution and a multivalued mapping. However the latter generation process may give basic possibility assignments that are not qualitative Moebius transforms (they may miss the monotonicity property over focal sets), but can be reduced to them while yielding the same capacity function.

One prospective use of the above results could be to represent capacities by small subsets of possibility measures (in contrast with $k$-maxitive capacities [8]). One interesting open problem is to find out, starting from a family of possibility distributions $\left\{\pi_{i}, i=1 \ldots, k\right\}$, how to derive the Moebius transform associated with $\gamma=\min _{i=1, \ldots, k} \Pi_{i}$ directly. Moreover the use of the maximal specific possibility distributions dominating a capacity could be instrumental in measuring its amount of uncertainty [11], for instance by comparing contour functions.

\section{References}

[1] G. Banon, Constructive decomposition of fuzzy measures in terms of possibility and necessity measures. Proc. VIth IFSA World Congress, São Paulo, Brazil, vol. I, p. 217-220, 1995.

[2] A. P. Dempster. Upper and lower probabilities induced by a multivalued mapping. Annals of Mathematical Statistics, 38:325-339, 1967.

[3] D. Dubois, H. Fargier. Making Discrete Sugeno Integrals More Discriminant, Int. J. of Approximate Reasoning, 50, 2009, 880-898

[4] D. Dubois, H. Fargier. Capacity refinements and their application to qualitative decision evaluation. In C. Sossai, G. Chemello (Eds.) Symbolic and Quantitative Approaches to Reasoning with Uncertainty (ECSQARU 2009), Verona (Italy), LNAI 5590, pages 311-322, Springer, 2009.

[5] D. Dubois, H. Prade. Upper and lower possibilities induced by a multivalued mapping, Proc. IFAC Symp. on Fuzzy Information, Knowledge Representation and Decision Analysis, Marseille, Jul. 19-21, 1983, 174-152.

[6] D. Dubois, H. Prade. Evidence measures based on fuzzy information, Automatica, 21, 547-562, 1985

[7] D. Dubois, H. Prade: Aggregation of possibility measures, In J. Kacprzyk, M. Fedrizzi (eds.): Multiperson Decision Making using Fuzzy Sets and Possibility Theory, Kluwer, Dordrecht, 5563, 1990

[8] M. Grabisch. On the representation of $k$ decomposable measures Proc. 7th IFSA world Congress, vol. 1, pages 478-483, Prague, 1997.

[9] M. Grabisch. The Moebius transform on symmetric ordered structures and its application to capacities on finite sets. Discrete Mathematics, 287:17-34, 2004.

[10] J.-L. Marichal. On Sugeno integrals as an aggregation function. Fuzzy Sets and Systems, 114(3):347-365, 2000.

[11] J.-L. Marichal, Marc Roubens: Entropy of Discrete Fuzzy Measures. Int. J. of Uncertainty, Fuzziness and Knowledge-Based Systems 8(6): 625-640 (2000)

[12] R. Mesiar, $k$-order pan-discrete fuzzy measures, Proc. 7th IFSA world Congress, vol. 1, pages 488-490, Prague, 1997.

[13] G. Shafer. A Mathematical Theory of Evidence. Princeton University Press, Princeton, 1976.

[14] D. Schmeidler. Core of exact games I. . J. Math. Analysis and Appl., 40, 214-225, 1972.

[15] E. Tsiporkova, B. De Baets: A General Framework for Upper and Lower Possibilities and Necessities. Int. J. of Uncertainty, Fuzziness and Knowledge-Based Systems 6(1): 1-34, 1998.

[16] P. Walley. Statistical Reasoning with Imprecise Probabilities. Chapman and Hall, 1991.

[17] S.M.K. Wong, Y.Y. Yao, P. Bollman, and H.C. Burger. Axiomatization of qualitative belief structure. IEEE trans. Systems Man and $C y$ bern., 21:726- 734, 1993. 